• 研究报告・

$$
\begin{aligned}
& \text { 秦岭南坡陕西洋县辖区哺乳动物 } \\
& \text { 物种多样性的空间分布格局 }
\end{aligned}
$$

\begin{abstract}
摘要: 物种多样性的空间分布格局一直是生态学和生物地理学研究的一个热点问题。山地生态系统的生境异质性 和物种多样性高, 适合研究物种多样性空间分布格局及其相关机制。2016年11月至2017年11月, 本研究选取秦岭 南坡陕西洋县辖区作为研究区域, 采用样线法、红外相机法和笼捕/夹捕法, 系统分析了 8 目 21 科 48 种哺乳动物物种 多样性的空间分布格局。研究结果发现秦岭南坡洋县辖区哺乳动物物种丰富度的空间分布格局大致是中南部低, 北部和东部高; 物种多样性指数大致是中南部和北部低, 东部高。啮齿类动物和非啮齿类动物的空间分布格局存 在差异。哺乳动物物种丰富度和多样性指数的垂直分布格局都符合中峰模式, 但啮齿类动物和非啮齿类动物间存 在差异。最优线性模型结果表明，研究地区哺乳动物物种多样性的空间分布格局受到多种环境因素的共同影响。 其中, 年均温与物种多样性的相关性最强, 在 6 个最优线性模型中贡献都是最大。综上, 秦岭南坡洋县辖区中高海 拔区域的物种多样性较高, 应加强对中高海拔地区的保护, 以维系该区域较高的生物多样性。
\end{abstract}

关键词：秦岭; 哺乳动物; 物种多样性; 空间分布格局; 垂直分布格局

\title{
Spatial distribution patterns of mammal diversity in Yangxian County of Shaanxi Province on the southern slope of the Qinling Mountains
} \author{
Beijing 100101 \\ Research Academy of Environmental Sciences, Beijing 100012 \\ 4 School of Life Sciences, Huaibei Normal University, Huaibei, Anhui 235000 \\ 5 Foping National Nature Reserve, Foping, Shaanxi 723400
}

Wenbo Yan ${ }^{1,2}$, Shengnan $\mathrm{Ji}^{3}$, Lingying Shuai ${ }^{4}$, Leigang Zhao ${ }^{5}$, Dapeng Zhu ${ }^{5}$, Zhigao Zeng ${ }^{2 *}$

1 Shaanxi Key Laboratory of Bio-Resources, Shaanxi University of Technology, Hanzhong, Shaanxi 723001

2 Key Laboratory of Animal Ecology and Conservation Biology, Institute of Zoology, Chinese Academy of Sciences,

3 State Environmental Protection Key Laboratory of Regional Ecological Processes and Functions Assessment, Chinese

\begin{abstract}
Studies on spatial patterns of biodiversity are of great interest in ecology and biogeography. Mountain ecosystems with high habitat heterogeneity and rich biodiversity are ideal for the study of biodiversity distribution. From November 2016 to November 2017, line transect survey, camera-trapping and cage-trapping/snap-trapping were used to investigate the mammal diversity in Yangxian County of Shaanxi Province, which is located on the southern slope of the Qinling Mountains. A total of 48 mammal species belonging to 21 families and 8 orders were identified in the study area. The spatial distribution of mammal species richness was generally low in the midland and south and high in the north and east of the study area. The spatial distribution of mammal species diversity was generally low in the midland and south and north and high in the east of the study area. The spatial distribution of rodents and non-rodent mammals were different. The elevational distribution of mammal species richness and diversity were unimodal. The
\end{abstract}

收稿日期: 2018-10-06; 接受日期: 2019-02-13

* 通讯作者 Author for correspondence. E-mail: zengzhg@ioz.ac.cn 
elevational distribution of rodents and non-rodent mammals were also different. The best model suggests that patterns of mammal species diversity are governed by the integrated effects of different environmental predictors, with annual mean temperature being the most important. Mammal species diversity peaked at mid and high elevation area. To maintain high biodiversity in the region, we should sustainably strengthen protection of mid and high elevation areas.

Key words: Qinling Mountains; mammal; species diversity; spatial distribution; elevational distribution

物种多样性的空间分布格局一直是生态学和 生物地理学研究的一个热点问题, 也是保护生物学 确定多样性热点和保护重点的重要依据之一。物种 多样性的分布格局是指在一定地域中物种多样性 在三维空间的分布状态, 即在经度、纬度和海拔高 度(水下深度)的分异情况(吴永杰和雷富民, 2013)。 鉴于海拔梯度综合了温度、降雨量、气压等多种环 境因子的影响, 温度在海拔梯度上的变化速率又是 纬度梯度的1,000倍(Barry, 2008), 因此成为生态学 家研究物种多样性分布规律的重要关注点(郑智等, 2014c)。而在相对较小的空间尺度上, 山地生态系 统的海拔梯度落差大, 成为研究物种多样性空间分 布格局的理想场所 (McCain, 2005; Rowe et al, 2015)。

对于物种多样性空间分布格局的形成与维持 机制, 研究者们提出了多种假说和解释, 包括面积 假说(Rosenzweig \& Ziv, 1999)、气候稳定性假说 (Stevens, 1989)、能量假说(Hawkins et al, 2003)、生 境异质性假说 (Heaney, 2010) 和中域效应假说 (Colwell \& Lees, 2000)等。其中中域效应假说重点 探讨几何边界对物种多样性空间分布格局的影响, 预测在几何边界的中心区域将出现物种多样性的 峰值, 是解释物种多样性空间分布格局的重要理论, 得到了广泛关注(吴永杰和雷富民, 2013; 郑智等, 2014b; 胡一鸣等, 2018)。

秦岭是我国亚热带和暖温带的分界线, 也是中 国动物地理区划中古北界和东洋界的分界线(张荣 祖, 2011)。秦岭为我国重要的生态过渡带, 加之山 体高大, 地形复杂, 物种多样性极其丰富, 是我国 生物多样性分布的中心地区之一; 《中国生物多样 性保护战略与行动计划》(2011-2030)已将秦岭列为 生物多样性保护优先区域之一。目前秦岭地区物种 多样性空间分布格局和相关机制的研究仍十分有 限, 多数研究仅限于局部地区的单个生物类群, 且 主要集中在植物类群(如唐志尧等, 2004; 唐志尧和
柯金虎，2004; 刘金虎等，2011; 王宇超和王得祥， 2013)、鸟类 ${ }^{(1)}$ 、两栖爬行类(郑智等, 2014a, b, c)和 哺乳类(李晟等, 2014; Shuai et al, 2017)。陕西省洋县 辖区位于秦岭中段南坡, 海拔跨度从389 $\mathrm{m}$ 至3,071 $\mathrm{m}$, 境内有长青国家级自然保护区和朱噮国家级自 然保护区, 哺乳动物物种多样性丰富(何百锁, 2001; 何百锁等, 2016)。洋县辖区内人口密度和人为干扰 比较大, 因而动物多样性的空间分布受到较大影 响。本研究分析了秦岭地区人为干扰下的物种多样 性空间分布格局, 为探讨大尺度范围物种多样性空 间分布格局的普遍模式、环境影响因素和演化机制 等宏生态学研究提供数据支撑。

\section{材料与方法}

\section{1 研究区域}

陕西省洋县辖区位于 $107^{\circ} 11^{\prime}-108^{\circ} 33^{\prime} \mathrm{E}, 3^{\circ} 02^{\prime}-$ $33^{\circ} 43^{\prime} \mathrm{N}$ 之间, 东西宽约 $56 \mathrm{~km}$, 南北长约76 km, 总 面积 $3,206 \mathrm{~km}^{2}$ (图1)。昏人坪梁兴隆岭海拔最高 (3,071 m), 黄金峡镇白沙渡海拔最低(389 m)。研究 区域属暖温带向北亚热带过渡地区，气候温暖湿润， 冬无严寒, 夏无酷暑。年日照时数1,800-2,000 h, 年 均气温 $12-14{ }^{\circ} \mathrm{C}$, 年均降水量 $900-1,000 \mathrm{~mm}$, 无霜 期 $238 \mathrm{~d}$ 。洋县中南部的汉中盆地是主要人口居住区 域, 人口密度较高, 东部较低, 北部最低(图1)。

洋县境内除低海拔地区分布有农作物如水稻 (Oryza sativa)、小麦(Triticum aestivum) 和油菜 (Brassica campestris)外, 随海拔从低到高还分布有 常绿阔叶林、阔叶混交林、针阔混交林、针叶林、 亚高山草甸5种植被类型, 共有乔木树种72科152属 321种。其中国家重点保护植物有铁杉(Tsuga chinensis)、秦岭冷杉(Abies chensiensis)、巴山冷杉(A. fargesii)、红豆杉(Taxus chinensis)、银杏(Ginkgo biloba)、香樟(Cinnamomum camphora)等。根据洋

(1) 田凯 (2017) 鄂西北野生脊椎动物物种多样性及地理分布格局研究. 硕士学位论文, 湖北大学, 武汉. 
县县志记载, 境内有兽类 60 多种, 鸟类250余种, 两 栖爬行动物20多种, 鱼类18种。被称为秦岭四宝的 国家一级重点保护野生动物大熊猫 (Ailuropoda melanoleuca)、川金丝猴(Rhinopithecus roxellana)、 秦岭羚牛 (Budorcas bedfordi) 和朱噮 (Nipponia nippon) 在这里均有分布。

\section{2 物种调查方法}

根据生态环境部对全国10 km × $10 \mathrm{~km}$ 网格划 分规则, 本研究区域包括 42 个网格, 我们根据分层 取样的原则选择其中的 22 个作为调查网格(图1)。在 每个调查网格内分别采用样线法、红外相机法和样 带法调查哺乳动物物种多样性。

(1)样线法: 每个调查网格内设置长2-3 km、宽 $6 \mathrm{~m}$ 的调查样线 2 条。2个人一组, 观测时行进速度为 $1 \mathrm{~km} / \mathrm{h}$, 记录样线上遇到的大中型哺乳动物的实 体、足迹链、粪便、食迹、卧迹等, 并判断动物的 种类和个体数量。2016年11-12月、2017年3-4月、 7 月和 11 月共进行了 4 次调查, 样线的总长度为 $464.58 \mathrm{~km}$ 。
(2)红外相机法: 将每个调查网格划分成25个 $2 \mathrm{~km} \times 2 \mathrm{~km}$ 的小网格, 根据分层取样的原则选择小 网格布设相机，每个小网格中布设 1 台，每个调查 网格内共2台。将红外相机设置在动物痕迹(粪便、 足迹和遗落物等)较多的地点(如兽径、水源点等处), 相机固定于离地面50-80 cm 的树干上，镜头基本与 地面平行。记录相机放置的日期、GPS位点、海拔、 坡度、坡向、动物痕迹以及人为干扰等信息。红外 相机数据每 3 个月读取 1 次, 然后将相机移动到其他 的小网格内。红外相机的有效位点数为 141 个, 总工 作时长为13,871相机日。

(3)样带法: 在每个调查网格内设置1条样带, 样带上每隔 $5 \mathrm{~m}$ 布设 1 个捕鼠笼或鼠夹, 共布设 100 个, 连续调查 3 天, 每天检查上夹动物, 总计用 300 个笼(夹)捕日; 同时在样带上每隔 $5 \mathrm{~m}$ 布设 1 个陷阱 (桶), 共 15 个, 连续 3 天调查食虫类小型哺乳动物。 在2016年秋季(10-11月)及2017年夏秋季(8-10月), 分两次共对 22 个网格进行了 13,320 个笼(夹)捕日及 530 个陷阱日的调查。

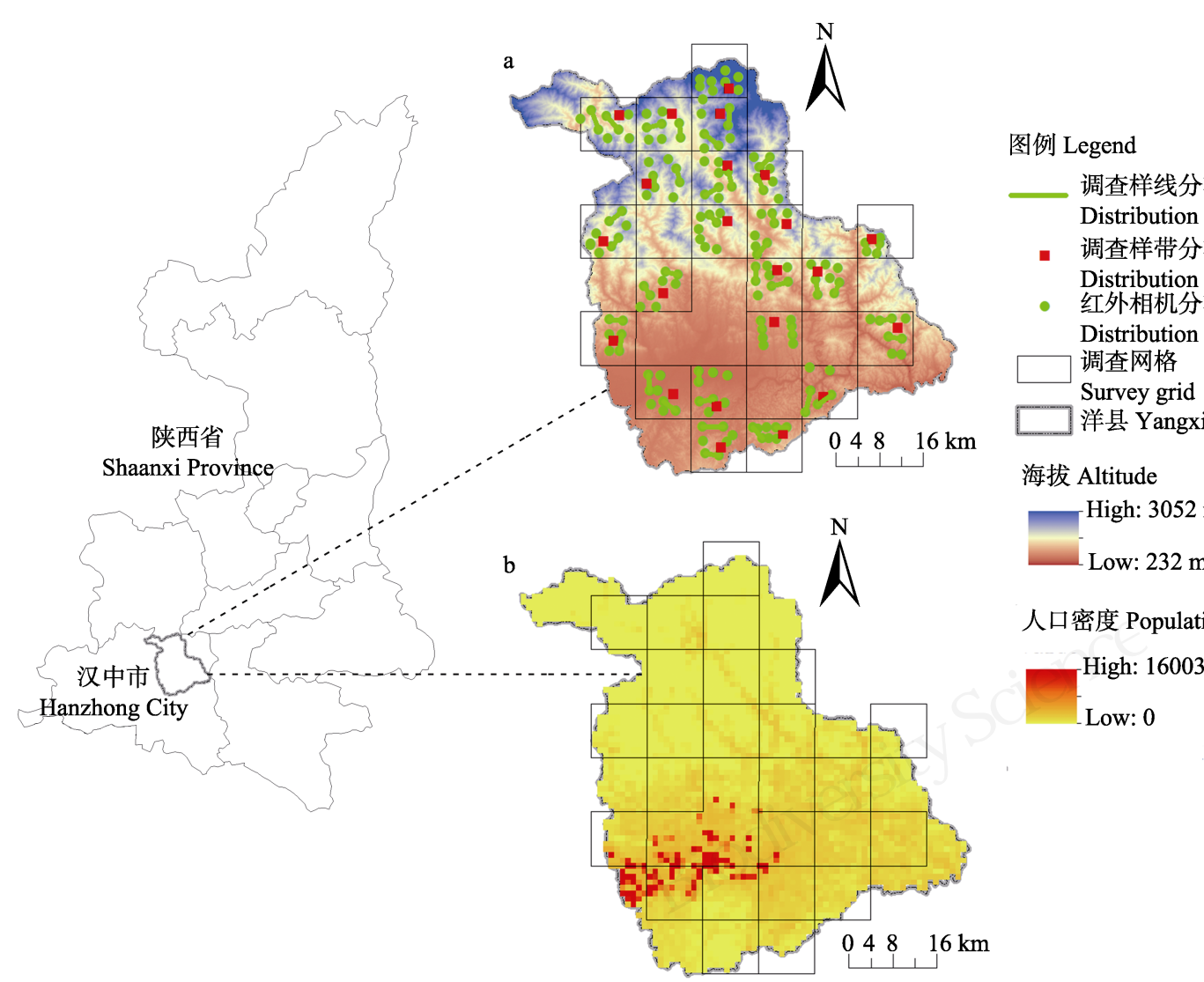

图1 研究地区的位置及调查设置(a)和人口分布(b)情况。数据来源于中国科学院资源环境科学数据中心(http://www.resdc.cn)。 Fig. 1 Location of study area, the status of survey settings (a) and population distribution (b). The data set is provided by Data Center for Resources and Environmental Sciences, Chinese Academy of Sciences (RESDC) (http://www.resdc.cn). 


\section{3 数据分析}

\subsection{1 物种多样性空间分布格局图的构建}

在ArcGIS 10.1软件中, 根据22个调查网格的物 种丰富度和多样性指数数据, 采用Kriging插值方法 构建研究地区物种丰富度和多样性指数的空间分 布格局图。物种丰富度采用物种的数目。物种多样 性采用Shannon-Wiener多样性指数, 计算公式如下:

$$
H^{\prime}=-\sum_{i=1}^{S} P_{i} \ln P_{i}
$$

式中, $S$ 为调查到的物种数; $P_{i}$ 为物种 $i$ 的个体数占 所有物种个体数的比例。

\subsection{2 物种多样性垂直分布模式的检验}

在ArcGIS 10.1软件中, 计算每个调查网格的平 均海拔。将每个调查网格的平均海拔作为自变量, 物种丰富度和多样性指数作为因变量, 构建多项式 回归模型, 并根据校正赤池信息准则AICc (the corrected Akaike information criterion)的最小值, 从一 阶和二阶的多项式(分别代表线性和单峰曲线)中选 出最优模型来确定物种丰富度和多样性指数的垂 直分布格局模式。海拔数据来源于https://earthexplorer. usgs.gov/网站，空间分辨率是 $30 \mathrm{~m} \times 30 \mathrm{~m}$ 。

\subsection{3 物种多样性与环境因子的关系检验}

在R 3.5.0 (R Development Core Team, 2018)中, 使用scatterplotMatrix函数分析环境因子与物种丰富 度和多样性指数的拟合曲线。根据拟合曲线分析结 果, 对每个环境因子变量增加二次项提高预测精 度。然后使用regsubsets函数进行全子集回归, 以校 正后的模型拟合优度(adjusted $R^{2}$ ) 为依据, 分别在 物种丰富度和多样性指数与环境因子的多元线性 回归模型中, 笁选出拟合优度最大的最优线性模 型。再使用 $\operatorname{lm}$ 函数拟合物种丰富度和多样性指数与 环境因子的多元线性回归模型, 并通过 $t$ 检验值比 较环境因子对模型的相对贡献大小。

本研究采用以下环境因子: 增强植被指数(enhanced vegetation index, EVI)、年均温(annual mean temperature, $\left.\mathrm{T}_{\text {mean }}\right) 、$ 年降水量(mean annual precipitation, AP)、植被类型数(vegetation number, Vegn)。 EVI数据选用2016年10月至2017年10月的MODIS数 据, 空间分辨率是 $250 \mathrm{~m} \times 250 \mathrm{~m}$, 来源于https:// earthexplorer.usgs.gov/网站。 $\mathrm{T}_{\text {mean }}$ AP和Vegn数据 的空间分辨率是 $1,000 \mathrm{~m} \times 1,000 \mathrm{~m}$, 来源于中国科 学院资源环境科学数据中心(http://www.resdc.cn)。 在ArcGIS 10.1软件中, 计算每个调查网格的EVI、 $\mathrm{T}_{\text {mean }}$ 和 $\mathrm{AP}$ 的平均值, 以及Vegn的数值。

\section{结果}

本次调查共鉴定哺乳动物 48 种(附录1), 隶属8 目 21 科 42 属, 其中啮齿类动物 6 科 17 属 22 种, 占哺 乳动物总种数的 $45.83 \%$ 。

\section{1 哺乳动物物种多样性的空间分布格局}

哺乳动物总体物种丰富度的空间分布格局大 致是中南部低, 北部和东部高(图2)。但是, 啮齿类 动物和非啮齿类动物的物种丰富度空间分布格局 存在差异: 啮齿类动物是中南部和北部低, 东部高; 而非啮齿类动物是中南部低, 北部高。哺乳动物总 体多样性指数的空间分布格局大致是中南部和北 部低, 东部高(图2)。啮齿类动物和非啮齿类动物的 物种多样性指数空间分布格局也存在差异: 啮齿类 动物是中南部和东部高, 北部低; 而非啮齿类动物 是中南部低, 北部和东部高。

\section{2 哺乳动物物种多样性的垂直分布格局}

哺乳动物总体物种丰富度垂直分布格局为正 中峰模式, 物种丰富度在海拔1,500 m左右最高(图 3a); 而总体物种多样性指数为左偏倚的中峰模式, 在海拔1,000 m左右最高(图3d)。啮齿动物的物种丰 富度和多样性指数垂直分布格局都为左偏倚的中 峰模式, 在海拔1,000 m左右最高(图3b, e)。非啮齿 类动物的物种丰富度垂直分布格局为右偏倚的中 峰模式, 物种丰富度在海拔1,800 m左右最高(图3c); 而物种多样性指数为正中峰模式, 在海拔 $1,500 \mathrm{~m}$ 左右最高(图3f)。

根据AICc最小值选出的最优多项式回归模型 都为二次方模型(表1), 即秦岭南坡洋县辖区哺乳动 物的物种多样性垂直分布格局都是单峰模式。

\section{3 影响哺乳动物物种多样性空间分布格局的环} 境因子

最优线性模型筛选结果显示由环境因子 $\mathrm{T}_{\text {mean }}$ 、 $\mathrm{T}_{\text {mean }}^{2} 、 V e g n 、 V e g n^{2} 、 E V I$ 和EVI ${ }^{2}$ 的组合模型对研究 地区哺乳动物总体物种丰富度空间分布格局的解释 率最高, 其中 $\mathrm{T}_{\text {mean }}^{2}$ EVI和 $E V I^{2}$ 具有显著影响(表2)。 $\mathrm{T}_{\text {mean }}^{2}$ 为负相关, 表明年均温对物种丰富度格局的影 响效果为倒钟型, 在中域温度区域具有最高的 

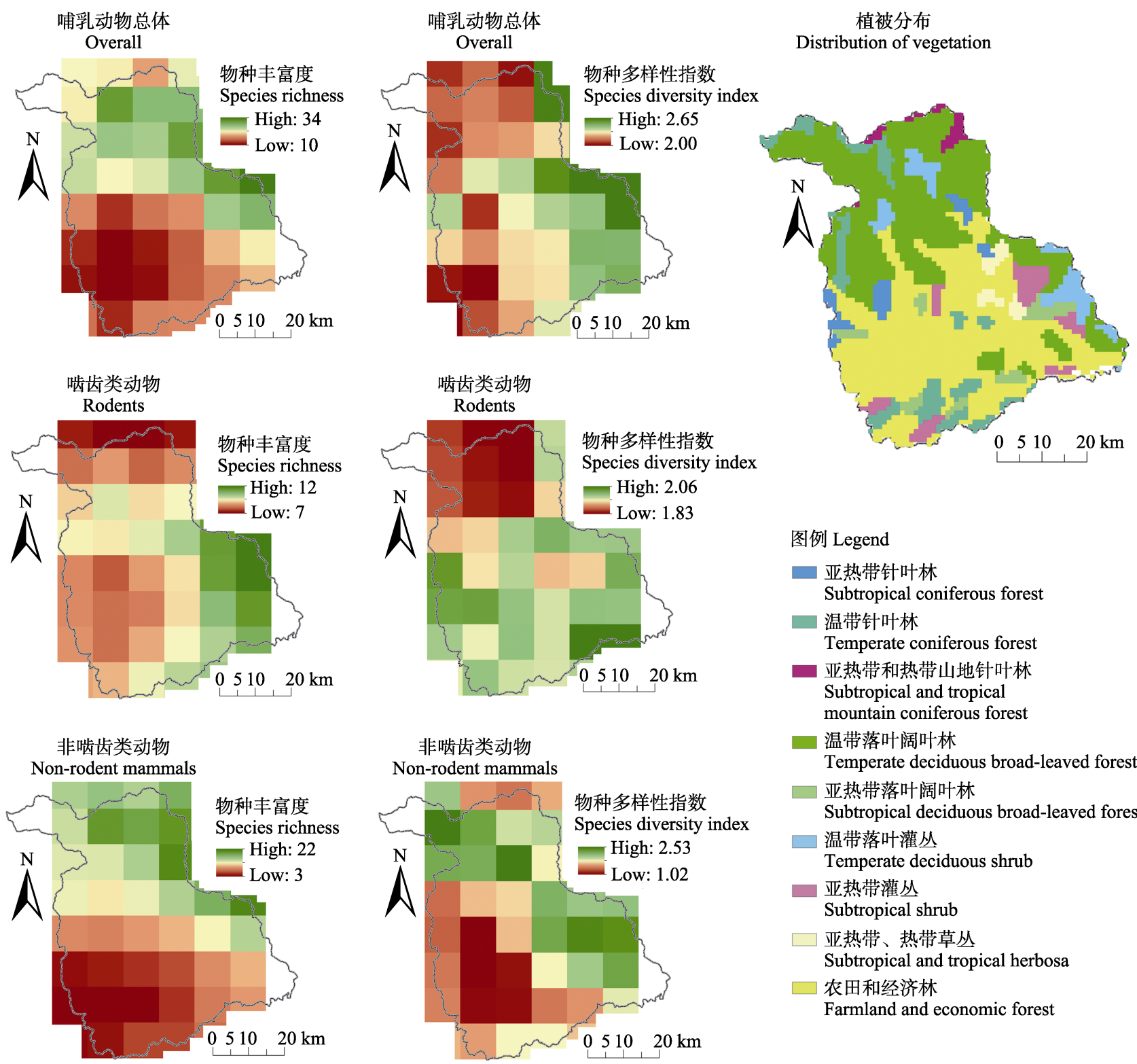
图例 Legend
亚热带针叶林
Subtropical coniferous forest
温带针叶林
Temperate coniferous forest
亚热带和热带山地针叶林
Subtropical and tropical
mountain coniferous forest
温带落叶阔叶林
Temperate deciduous broad-leaved forest
$\square$ 亚热带落叶阔叶林
Subtropical deciduous broad-leaved forest
温带落叶灌丛
Temperate deciduous shrub
亚热带灌丛
Subtropical shrub
亚热带、热带草丛
Subtropical and tropical herbosa
$\square$ 农田和经济林
Farmland and economic forest

图2 秦岭南坡洋县辖区哺乳动物物种多样性的空间分布格局

Fig. 2 Spatial distribution patterns of mammal species diversity in Yangxian County on the southern slope of the Qinling Mountains

物种丰富度。 $\mathrm{EVI}^{2}$ 为正相关, 表明增强植被指数对 物种丰富度格局的影响效果为钟型, 在中域增强植 被指数的区域具有较低的物种丰富度。 $\mathrm{EVI}^{2}$ 对模型 解释率的贡献最大, 表明增强植被指数对哺乳动物 总体物种丰富度空间分布格局起主导作用。由 $T_{\text {mean }} \mathrm{T}_{\text {mean }}^{2} \mathrm{AP} 、 \mathrm{AP}^{2}$ 和 Vegn ${ }^{2}$ 组成的模型对哺乳 动物总体多样性指数空间分布格局的解释率最高

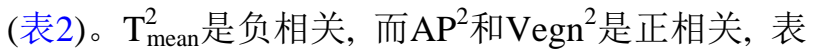
明年平均降水量和植被类型复杂性对物种多样性 指数格局的影响效果是钟型, 在中域年平均降水量
和植被类型复杂区域具有最低的物种多样性指数。 $\mathrm{AP}^{2}$ 对模型解释率的贡献最大, 表明年平均降水量 对哺乳动物总体物种多样性指数空间分布格局起 主导作用。

最优线性模型篮选结果显示, $\mathrm{T}_{\text {mean }} 、 \mathrm{~T}_{\text {mean }}^{2} \mathrm{AP}^{2} 、$ Vegn、EVI和EVI ${ }^{2}$ 的组合对啮齿类动物的物种丰富 度空间分布格局解释率最高(表2)。 $\mathrm{T}_{\text {mean }}^{2}$ 为负相关, 而 $\mathrm{AP}^{2}$ 和 $\mathrm{EVI}^{2}$ 是正相关。物种丰富度与植被类型数 显示为正相关关系。 $\mathrm{T}_{\text {mean }}$ 对模型解释率的贡献最大, 表明年均温对啮齿类动物的物种丰富度空间分布 

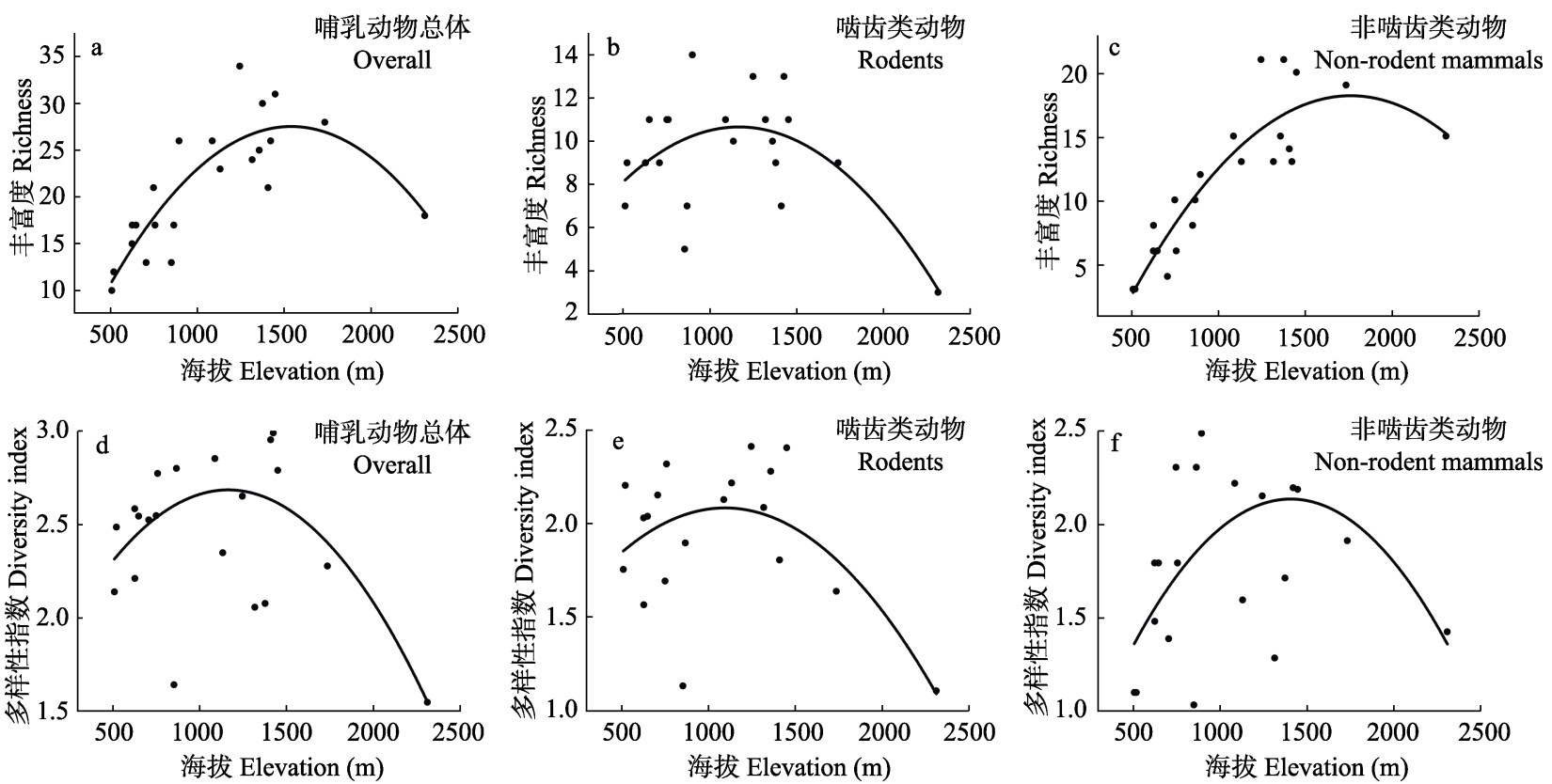

图3 秦岭南坡洋县辖区哺乳动物物种多样性的垂直分布格局

Fig. 3 Elevational distribution patterns of mammal species diversity in Yangxian County on the southern slope of the Qinling Mountains

表1 秦岭南坡洋县辖区哺乳动物物种多样性垂直分布的多项式回归

Table 1 Polynomial regressions for the mammal species diversity along the elevational gradients in Yangxian County on the southern slope of the Qinling Mountains

\begin{tabular}{llll}
\hline $\begin{array}{l}\text { Sp物种分组 } \\
\text { Species groups }\end{array}$ & 多样性 & $\begin{array}{l}\text { 校正赤池信息准则模型差值(一阶) } \\
\text { Corrected Akaike information criterion } \\
\text { (Order 1) }\end{array}$ & $\begin{array}{l}\text { 校正赤池信息准则模型差值(二阶) } \\
\text { Corrected Akaike information criterion } \\
\text { (Order 2) }\end{array}$ \\
\hline 哺乳动物总体 & 丰富度 Richness & 16.82 & 0 \\
Overall & 多样性指数 Diversity index & 5.06 & 0 \\
啮齿类动物 & 丰富度 Richness & 7.82 & 0 \\
Rodents & 多样性指数 Diversity index & 1.66 & 0 \\
非啮齿类动物 & 丰富度 Richness & 14.46 & 0 \\
Non-rodent mammals & 多样性指数 Diversity index & 5.13 & 0 \\
\hline
\end{tabular}

格局起主导作用。由 $\mathrm{T}_{\text {mean }} 、 \mathrm{~T}_{\text {mean }}^{2} 、 \mathrm{AP}^{2} 、 \mathrm{Vegn}^{2} 、 \mathrm{EVI}$ 和 $\mathrm{EVI}^{2}$ 组成的模型对啮齿类动物的物种多样性指 数空间分布格局解释率最高, 但仅 $\mathrm{T}_{\text {mean 具有显著影 }}$ 响(表2), 且呈正相关关系。

最优线性模型篎选结果显示, $\mathrm{T}_{\text {mean }}^{2}$ V Vegn、 $\mathrm{Vegn}^{2}$ 和 $\mathrm{EVI}^{2}$ 的组合对非齿齿类动物的物种丰富度 空间分布格局的解释率最高, 其中 $\mathrm{T}_{\text {mean }}^{2}$ 和 $\mathrm{EVI}^{2}$ 具有 显著影响(表2)。 $T_{\text {mean }}^{2}$ 为负相关, $E^{2} I^{2}$ 为正相关; $T_{\text {mean }}^{2}$ 对模型解释率的贡献最大。 $\mathrm{T}_{\text {mean }} 、 \mathrm{~T}_{\text {mean }}^{2} 、 \mathrm{Vegn}^{2}$ 和 $\mathrm{EVI}^{2}$ 的组合对非齿齿类动物的物种多样性指数空 间分布格局的解释率最高, 其中 $\mathrm{T}_{\text {mean }} 、 \mathrm{~T}_{\text {mean }}^{2}$ 和 $\mathrm{Vegn}^{2}$ 具有显著影响(表2)。 $T_{\text {mean }}^{2}$ 为负相关; $V \operatorname{Vgn}^{2}$ 是正相关， 且对模型解释率的贡献最大。

\section{3 讨论}

由于取样困难、数据难于获得等原因, 目前关 于哺乳动物物种多样性空间分布格局的研究主要 集中于物种丰富度方面(McCain, 2005; 王新建等, 2007; 林金等, 2009; Hu et al, 2017), 而关于物种多 样性指数的研究相对较少(吴永杰和雷富民, 2013)。 本研究综合采用了样线法、红外相机法和笼捕/夹捕 法, 不仅获得哺乳动物的物种数, 还调查到每物种 的数量, 因而不仅能探讨哺乳动物物种丰富度的空 间分布格局, 也能研究其物种多样性指数状况。本 次调查共确认分布 48 种哺乳动物, 其中 20 种为大中 型哺乳动物(包括灵长类、食肉类和有蹄类; 曾治高 
表2 秦岭南坡洋县辖区哺乳动物物种多样性与环境因子最 优线性模型

Table 2 Determinants of species diversity for mammals from best-fit explanatory models in Yangxian County on the southern slope of the Qinling Mountains

\begin{tabular}{|c|c|c|c|c|}
\hline $\begin{array}{l}\text { 多样性 } \\
\text { Diversity }\end{array}$ & $\begin{array}{l}\text { 环境因子 } \\
\text { Environment } \\
\text { variables }\end{array}$ & $\begin{array}{l}\text { 因子系数 } \\
\text { Coefficient }\end{array}$ & $t$ & $P$ \\
\hline \multicolumn{5}{|c|}{ 哺乳动物总体 Overall } \\
\hline \multirow{6}{*}{$\begin{array}{l}\text { 丰富度 } \\
\text { Richness }\end{array}$} & $\mathrm{T}_{\text {mean }}$ & 5.71 & 1.63 & 0.12 \\
\hline & $\mathrm{T}_{\text {mean }}^{2}$ & -0.32 & -2.14 & $<0.05$ \\
\hline & Vegn & 8.96 & 2.09 & 0.05 \\
\hline & $\operatorname{Vegn}^{2}$ & -0.99 & -1.71 & 0.11 \\
\hline & EVI & $-2.38 \mathrm{e}+03$ & -2.17 & $<0.05$ \\
\hline & $\mathrm{EVI}^{2}$ & $3.62 \mathrm{e}+03$ & 2.25 & $<0.05$ \\
\hline \multirow{5}{*}{$\begin{array}{l}\text { 多样性指数 } \\
\text { Diversity index }\end{array}$} & $\mathrm{T}_{\text {mean }}$ & 1.02 & 2.55 & $<0.05$ \\
\hline & $\mathrm{T}_{\text {mean }}^{2}$ & $-4.73 e-02$ & -2.21 & $<0.05$ \\
\hline & $\mathrm{AP}$ & -0.16 & -2.31 & $<0.05$ \\
\hline & $\mathrm{AP}^{2}$ & $9.05 \mathrm{e}-05$ & 2.34 & $<0.05$ \\
\hline & $\operatorname{Vegn}^{2}$ & $2.59 \mathrm{e}-02$ & 2.28 & $<0.05$ \\
\hline \multicolumn{5}{|c|}{ 啮齿类动物 Rodents } \\
\hline \multirow{6}{*}{$\begin{array}{l}\text { 丰富度 } \\
\text { Richness }\end{array}$} & $\mathrm{T}_{\text {mean }}$ & 7.11 & 3.12 & $<0.01$ \\
\hline & $\mathrm{T}_{\text {mean }}^{2}$ & -0.33 & -3.10 & $<0.01$ \\
\hline & $\mathrm{AP}^{2}$ & $2.60 \mathrm{e}-05$ & 2.36 & $<0.05$ \\
\hline & Vegn & 1.04 & 2.73 & $<0.05$ \\
\hline & EVI & $-1.45 \mathrm{e}+03$ & -2.68 & $<0.05$ \\
\hline & $\mathrm{EVI}^{2}$ & $2.10 \mathrm{e}+03$ & 2.70 & $<0.05$ \\
\hline \multirow{6}{*}{$\begin{array}{l}\text { 多样性指数 } \\
\text { Diversity index }\end{array}$} & $\mathrm{T}_{\text {mean }}$ & 1.43 & 2.13 & $<0.05$ \\
\hline & $\mathrm{T}_{\text {mean }}^{2}$ & $-6.41 \mathrm{e}-02$ & -2.06 & 0.06 \\
\hline & $\mathrm{AP}^{2}$ & 3.79e-06 & 1.19 & 0.25 \\
\hline & $\operatorname{Vegn}^{2}$ & $1.85 \mathrm{e}-02$ & 1.24 & 0.24 \\
\hline & EVI & $-2.62 \mathrm{e}+02$ & -1.66 & 0.12 \\
\hline & $\mathrm{EVI}^{2}$ & $3.66 \mathrm{e}+02$ & 1.61 & 0.13 \\
\hline \multicolumn{5}{|c|}{ 非啮齿类动物 Non-rodent mammals } \\
\hline \multirow{4}{*}{$\begin{array}{l}\text { 丰富度 } \\
\text { Richness }\end{array}$} & $\mathrm{T}_{\text {mean }}^{2}$ & -0.09 & -5.81 & $<0.01$ \\
\hline & Vegn & 5.97 & 1.92 & 0.07 \\
\hline & $\operatorname{Vegn}^{2}$ & -0.74 & -1.79 & 0.09 \\
\hline & $\mathrm{EVI}^{2}$ & $1.84 \mathrm{e}+02$ & 3.22 & $<0.01$ \\
\hline \multirow{4}{*}{$\begin{array}{l}\text { 多样性指数 } \\
\text { Diversity index }\end{array}$} & $\mathrm{T}_{\text {mean }}$ & 0.94 & 2.35 & $<0.05$ \\
\hline & $\mathrm{T}_{\text {mean }}^{2}$ & -0.04 & -2.47 & $<0.05$ \\
\hline & $\operatorname{Vegn}^{2}$ & 0.04 & 3.19 & $<0.01$ \\
\hline & $\mathrm{EVI}^{2}$ & -16.74 & -1.49 & 0.16 \\
\hline
\end{tabular}

EVI: 增强植被指数; $\mathrm{T}_{\text {mean }}$ ：年均温; $\mathrm{AP}:$ 年降水量; Vegn: 植被类型 数。

EVI, Enhanced vegetation index; $\mathrm{T}_{\text {mean }}$, Annual mean temperature; AP, Mean annual precipitation; Vegn, Vegetation number.

等, 2006)。何百锁(2001)经1997-2000年的野外调查 确认长青国家级自然保护区内分布 52 种哺乳动物。
何百锁等(2016)基于红外相机确定长青国家级自然 保护区内分布17种大中型哺乳动物。可见，本调查 结果基本反映了研究地区的哺乳动物群落组成。

人类活动干扰对研究地区哺乳动物物种多样 性的空间分布格局具有较大影响。在人口密度最高 的中南部地区，哺乳动物总体物种丰富度和多样性 指数都最低(图1, 2)。物种丰富度和多样性指数的空 间分布格局在啮齿类动物和非啮齿类动物之间存 在差异。啮齿类动物在人口密度较小的东部地区丰 富度最高, 而非啮齿类动物则在人口密度最小的北 部地区高。由于边缘效应，适度人类干扰生境中小 型啮齿类动物的物种多样性可能最高(肖治术等, 2002)。据此推测，可能是近年来的天然林保护工程 和移民搬迁工程，使洋县东部地区的次生林和灌木 林受到的干扰减弱，且出现了大量的弃耕地，隐蔽 条件较好, 导致森林型鼠类和农田型鼠类多在此处 出没, 前者如大林姬鼠(Apodemus peninsulae)、齐氏 姬鼠(A. chevrieri)和白腹巨鼠(Leopoldamys edwardsi), 后者如大仓鼠(Tscheskia triton)、北社鼠(Niviventer confucianus)。因此, 适度人类干扰的东部地区啮齿 类动物的物种丰富度和多样性指数最高。而大中型 野生动物对人类活动干扰敏感(张跃等, 2012), 其物 种丰富度和多样性指数在人口密度最高的中南部 地区最低，而在人口密度最小的北部地区最高。

秦岭南坡洋县辖区不同生物类群的物种丰富 度垂直分布格局不同：哺乳动物总体符合正中峰模 式，啮齿类动物符合左偏倚的中峰模式，非啮齿类 动物符合右偏倚的中峰模式。这一结果与秦岭太白 山南坡齿齿类动物物种丰富度随海拔升高而呈现 单调递减模式存在一定的差异(Shuai et al, 2017), 其原因可能是本研究的海拔跨度从389 m至3,071 m, 啮齿类动物物种丰富度在海拔 $1,000 \mathrm{~m}$ 左右最高, 而Shuai等(2017)研究的秦岭南坡最低起始海拔是 $1,200 \mathrm{~m}$, 可能不足以展示出中峰模式。李晟等(2014) 的研究显示洋县辖区长青国家级自然保护区内有 蹄类动物物种丰富度的垂直分布格局呈现中峰模 式, 本研究结果与之相似。不同物种类群分布格局 的差异受各自格局潜在环境因素的影响(Wu et al, 2013)。本研究中啮齿类动物的空间分布格局受到年 均温、降水量、增强植被指数和植被类型数等环境 因子的显著影响，而非啮齿类动物只受到年均温和 增强植被指数的显著影响。 
秦岭南坡洋县辖区哺乳动物的物种多样性与 丰富度的垂直分布格局存在差异。Shannon-Wiener 多样性指数相比物种丰富度更能解释物种分布的 均匀度(Spellerberg, 2008)。因此, Shannon-Wiener多 样性指数对常见种敏感, 而物种丰富度对稀有种敏 感(马克平和刘玉明, 1994)。野猪(Sus scrofa)和小鹿 (Muntiacus reevesi) 等常见种在本研究地区主要分 布在中低海拔, 而大熊猫、川金丝猴和秦岭羚牛等 稀有种主要分布在较高海拔。所以, 哺乳动物总体 和非啮齿类动物的物种多样性指数垂直分布格局 相比物种丰富度表现出向低海拔偏倚的模式。影响 物种多样性指数空间分布格局的环境因子与物种 丰富度也存在明显差异。

秦岭南坡洋县辖区哺乳动物物种多样性的空 间分布格局受到多种环境因素的共同影响。其中, 年均温与物种多样性的相关性最强, 在 6 个最优线 性模型中贡献最大。根据物种多样性地理格局的环 境热量假说, 温度越高, 越有利于促进物种分化并 提高物种多样性(Currie, 1991)。但是, 本研究结果 显示温度对物种多样性的影响呈现中峰模式, 这与 环境热量假说存在差异。这种差异可能是由于高山 生态系统虽然随海拔变化形成的环境差异与纬度 相似, 但是还是存在一定的差异, 而环境热量假说 是基于纬度的地理差异提出的(Currie, 1991)。

综上, 秦岭南坡洋县辖区哺乳动物物种多样性 的空间分布格局受到人为干扰、海拔、温度等多种 因素的影响。北部和东部的中高海拔地区具有较高 的物种多样性, 为此, 应加强对洋县辖区中高海拔 地区的保护，以维系该区域较高的生物多样性。

致谢: 感谢参与野外动物调查工作的巩会生、段延、 刘红红、王子星、李冬森、杜晨、李凯亮、王江涛、 李林宇等多位人员, 特别感谢巩会生帮助制作哺乳 动物标本。

\section{参考文献}

Barry RG (2008) Mountain Weather and Climate. Cambridge University Press, Cambridge.

Colwell RK, Lees DC (2000) The mid-domain effect: Geometric constraints on the geography of species richness. Trends in Ecology \& Evolution, 15, 70-76.

Currie DJ (1991) Energy and large-scale patterns of animaland plant-species richness. The American Naturalist, 137,
27-49.

Hawkins BA, Field R, Cornell HV, Currie DJ, Kaufman DM, Kerr JT, Mittelbach GG, Oberdorff T, O’Brien EM (2003) Energy, water, and broad-scale geographic patterns of species richness. Ecology, 84, 3105-3117.

He BS (2001) Animal resources in Changqing Nature Reserve. Sichuan Journal of Zoology, 20(3), 132-134. (in Chinese) [何百锁 (2001) 长青自然保护区的兽类资源. 四川动物, 20(3), 132-134.]

He BS, Sun RQ, Chen P, Dong W, Wang J, Wang DJ, Li S (2016) Baseline survey of mammal and bird diversity using camera-trapping in the Changqing National Nature Reserve of Shaanxi Province. Acta Theriologica Sinica, 36, 348-356. (in Chinese with English abstract) [何百锁, 孙瑞谦, 陈鹏, 董伟, 王军, 王大军, 李晟 (2016) 基于红外相机技术调 查长青国家级自然保护区兽类和鸟类多样性. 兽类学报, 36, 348-356.]

Heaney LR (2010) Small mammal diversity along elevational gradients in the Philippines: An assessment of patterns and hypotheses. Global Ecology \& Biogeography, 10, 15-39.

Hu YM, Jin K, Huang ZW, Ding ZF, Liang JC, Pan XY, Hu HJ, Jiang ZG (2017) Elevational patterns of non-volant small mammal species richness in Gyirong Valley, Central Himalaya: Evaluating multiple spatial and environmental drivers. Journal of Biogeography, 44, 2764-2777.

Hu YM, Liang JC, Jin K, Ding ZF, Zhou ZX, Hu HJ, Jiang ZG (2018) The elevational patterns of mammalian richness in the Himalayas. Biodiversity Science, 26, 191-201. (in Chinese with English abstract) [胡一鸣, 梁健超, 金芘, 丁 志锋，周智金金，胡慧建，蒋志刚 (2018) 喜马拉雅山哺乳 动物物种多样性垂直分布格局. 生物多样性, 26, 191-201.]

Li S, Zhang XF, Chen P, Wang J, Xiang DQ, Dong W, Zhang XM, He BS, Sun RQ, Zhao NX, Wang DJ (2014) The community structure and elevational patterns of forest ungulates at the southern slope of the Qinling Mountains, China. Chinese Journal of Zoology, 49, 633-643. (in Chinese with English abstract) [李晟, 张晓峰, 陈鹏, 王军, 向定乾，董伟，张希明，何百锁，孙瑞谦，赵纳勋，王大 军 (2014) 秦岭南坡森林有蹄类群落组成与垂直分布特 征. 动物学杂志, 49, 633-643.]

Lin X, Wang ZH, Tang ZY, Zhao SQ, Fang JY (2009) Geographic patterns and environmental correlates of terrestrial mammal species richness in China. Biodiversity Science, 17, 652-663. (in Chinese with English abstract) [林 金鍂，王志恒，唐志尧，赵淑清，方精云 (2009) 中国陆栖 哺乳动物物种丰富度的地理格局及其与环境因子的关系. 生物多样性, 17, 652-663.]

Liu JH, Wang DX, Wang YC, Hu YN (2011) Changes in plant species diversity of natural coniferous forests along the altitudinal gradient in the Youshui River, Qinling Mountains. Journal of Northwest Forestry University, 26(3), 6-11. (in Chinese with English abstract) [刘金虎, 王得祥, 王宇超, 胡有宁 (2011) 秦岭西水河天然针叶林物种多 样性的垂直格局. 西北林学院学报, 26(3), 6-11.]

Ma KP, Liu YM (1994) Measurement of biotic community diversity. I. $\alpha$ diversity (Part 2). Chinese Biodiversity, 2, 231-239. (in Chinese) [马克平, 刘玉明 (1994) 生物群落 
多样性的测度方法. I. $\alpha$ 多样性的测度方法(下). 生物多样 性, 2, 231-239.]

McCain CM (2005) Elevational gradients in diversity of small mammals. Ecology, 86, 366-372.

Rosenzweig ML, Ziv Y (1999) The echo pattern of species diversity: Pattern and processes. Ecography, 22, 614-628.

Rowe RJ, Heaney LR, Rickart EA (2015) Scale effects on the pattern and predictors of small mammal diversity along a local elevational gradient in the Great Basin. Journal of Biogeography, 42, 1964-1974.

Shuai LY, Ren CL, Yan WB, Song YL, Zeng ZG (2017) Different elevational patterns of rodent species richness between the southern and northern slopes of a mountain. Scientific Reports, 7, 8743.

Spellerberg IF (2008) Shannon-Wiener index. In: Encyclopedia of Ecology (eds Jørgensen SE, Fath BD), pp. 3249-3252. Academic Press, Oxford.

Stevens GC (1989) The latitudinal gradient in geographical range: How so many species coexist in the tropics. The American Naturalist, 133, 240-256.

Tang ZY, Fang JY, Zhang L (2004) Patterns of woody plant species diversity along environmental gradients on Mt. Taibai, Qinling Mountains. Biodiversity Science, 12, 115-122. (in Chinese with English abstract) [唐志尧, 方精 云, 张玲 (2004) 秦岭太白山木本植物物种多样性的梯 度格局及环境解释. 生物多样性, 12, 115-122.]

Tang ZY, Ke JH (2004) Altitudinal patterns of plant species diversity in Mt. Niubeiliang, Qinling Mountains. Biodiversity Science, 12, 108-114. (in Chinese with English abstract) [唐志尧, 柯金虎 (2004) 秦岭牛背梁植物物种 多样性垂直分布格局. 生物多样性, 12, 108-114.]

Wang XJ, Zhou LZ, Zhang YY, Xing YJ, Gu CM (2007) Distribution patterns and species diversity of mammals in Anhui Province. Acta Theriologica Sinica, 27, 175-184. (in Chinese with English abstract) [王新建, 周立志, 张有瑜, 邢雅俊, 顾长明 (2007) 安徽省兽类物种多样性及其分 布格局. 兽类学报, 27, 175-184.]

Wang YC, Wang DX (2013) Study on elevational patterns of plant species diversity and community complexity in Foping Natural Reserve. Acta Botanica Boreali-Occidentalia Sinica, 33, 169-176. (in Chinese with English abstract) [王宇超, 王得祥 (2013) 佛坪自然保护区植物群落物种多样性和 复杂性的海拔格局研究. 西北植物学报, 33, 169-176.]

Wu YJ, Lei FM (2013) Species richness patterns and mechanisms along the elevational gradients. Chinese Journal of Zoology, 48, 797-807. (in Chinese with English abstract) [吴永杰, 雷富民 (2013) 物种丰富度垂直分布格局及影 响机制. 动物学杂志, 48, 797-807.]

Wu YJ, Yang QS, Wen ZX, Xia L, Zhang Q, Zhou HM (2013) What drives the species richness patterns of non-volant small mammals along a subtropical elevational gradient?
Ecography, 36, 185-196.

Xiao ZS, Wang YS, Zhang ZB, Ma Y (2002) Preliminary studies on the relationships between communities of small mammals and habitat types in Dujiangyan Region, Sichuan. Biodiversity Science, 10, 163-169. (in Chinese with English abstract) [肖治术, 王玉山, 张知涁, 马勇 (2002) 都江堰 地区小型哺乳动物群落与生境类型关系的初步研究. 生 物多样性, 10, 163-169.]

Zeng ZG, Gong HS, Song YL, Miao T, Ma SR (2006) Resource, fauna and ecological distribution of large- and medium-sized mammals in Majiashan Nature Reserve, Shaanxi. Sichuan Journal of Zoology, 25(1), 87-91. (in Chinese with English abstract) [曾治高, 巩会生, 宋延龄, 缪涛, 马顺荣 (2006) 陕西马家山自然保护区大中型兽 类的资源及区系与生态分布. 四川动物, 25(1), 87-91.]

Zhang RZ (2011) Zoogeography of China. Science Press, Beijing. (in Chinese) [张荣祖 (2011) 中国动物地理. 科学 出版社, 北京.]

Zhang Y, Lei KM, Zhang YK, Xiao CL, Yang YH, Sun HO, Li SJ (2012) Effects of vegetation, elevation and human disturbance on the distribution of large- and medium-sized wildlife: A case study in Jiuzhaigou Nature Reserve. Acta Ecologica Sinica, 32, 4228-4235. (in Chinese with English abstract) [张跃, 雷开明, 张语克, 肖长林, 杨玉花, 孙鸿 鸥，李淑君 (2012) 植被、海拔、人为干扰对大中型野生 动物分布的影响——九寨沟自然保护区为例. 生态学 报, 32, 4228-4235.]

Zheng Z, Gong DJ, Sun CX (2014a) Elevational pattern of species richness and species range size of herpetofauna in Baishuijiang Nature Reserve: A test of Rapoport's rule. Chinese Journal of Ecology, 33, 537-546. (in Chinese with English abstract) [郑智, 龚大洁, 孙呈祥 (2014a) 白水江 自然保护区两栖爬行动物物种丰富度和种域海拔梯度格 局及对Rapoport法则的验证. 生态学杂志, 33, 537-546.]

Zheng Z, Gong DJ, Sun CX, Li XJ, Li WJ (2014b) Elevational pattern of amphibian and reptile diversity in Qinling range and explanation. Biodiversity Science, 22, 596-607. (in Chinese with English abstract) [郑智, 龚大洁, 孙呈祥, 李 晓军, 李万江 (2014b) 秦岭两栖、爬行动物物种多样性 海拔分布格局及其解释. 生物多样性, 22, 596-607.]

Zheng Z, Gong DJ, Zhang Q (2014c) Explanation of elevational patterns of amphibian and reptile species richness in the Baishuijiang Nature Reserve: Water-energy dynamic hypothesis and habitat heterogeneity hypothesis. Chinese Journal of Ecology, 33, 3332-3338. (in Chinese with English abstract) [郑智, 龚大洁, 张乾 (2014c) 白水 江自然保护区两栖、爬行动物物种丰富度的海拔梯度格 局的解释: 水分能量动态假设和生境异质性假设. 生态 学杂志, 33, 3332-3338.]

(责任编委: 李晟 责任编辑: 间文杰)

\section{附录 Supplementary Material}

\section{附录1 秦岭南坡陕西洋县辖区的哺乳动物名录}

Appendix 1 List of mammal species in Yangxian County of Shaanxi Province on the southern slope of the Qinling Mountains http://www.biodiversity-science.net/fileup/PDF/2018266-1.pdf 
颜文博, 吉晟男, 帅凌鹰, 赵雷刚, 朱大鹏, 曾治高. 秦岭南坡陕西洋县辖区哺乳动物物种多样性的空间分 布格局. 生物多样性, 2019, 27 (2): 177-185.

http://www.biodiversity-science.net/CN/10.17520/biods.2018266

附录1 秦岭南坡陕西洋县辖区的哺乳动物名录

Appendix 1 List of mammal species in Yangxian County of Shaanxi Province on the southern slope of the Qinling Mountains

\begin{tabular}{|c|c|c|c|c|c|}
\hline 中文名 & 学名 & 目 & 科 & 保护等级 & 记录方式 \\
\hline Chinese name & Latin name & Order & Family & $\begin{array}{l}\text { Protection } \\
\text { level }\end{array}$ & Recording \\
\hline 东北刺猬 & Erinaceus amurensis & Erinaceomorpha & Erinaceidae & & 样线、夹捕 \\
\hline 林猬 & Mesechinus hughi & Erinaceomorpha & Erinaceidae & & 样线、夹捕 \\
\hline 陕西鼠鼣 & Sorex sinalis & Soricomorpha & Soricidae & & 样线、夹捕 \\
\hline 马铁菊头蝠 & Rhinolophus ferrumequinum & Chiroptera & Rhinolophidae & & 样线 \\
\hline 普通伏翼 & Pipistrellus pipistrellus & Chiroptera & Vesperitilionidae & & 样线 \\
\hline 中华山蝠 & Nyctalus plancyi & Chiroptera & Vesperitilionidae & & 样线 \\
\hline 川金丝猴 & Rhinopithecus roxellana & Primates & Cercopithecidae & I & 红外相机 \\
\hline 貉 & Nyctereutes procyonoides & Carnivora & Canidae & & 红外相机 \\
\hline 黑熊 & Selenarctos thibetanus & Carnivora & Ursidae & II & 红外相机 \\
\hline 大熊猫 & Ailuropoda melanoleuca & Carnivora & Ailuropodidae & I & 红外相机、样线 \\
\hline 黄喉貂 & Martes flavigula & Carnivora & Mustelidae & II & 红外相机 \\
\hline 黄鼠由 & Mustela sibirica & Carnivora & Mustelidae & & 红外相机、样线 \\
\hline 鼠由獾 & Melogale moschata & Carnivora & Mustelidae & & 红外相机、样线 \\
\hline 猪獾 & Arctonyx collaris & Carnivora & Mustelidae & & 红外相机、样线 \\
\hline 水獭 & Lutra lutra & Carnivora & Mustelidae & II & 红外相机 \\
\hline 果子狸 & Paguma larvata & Carnivora & Viverridae & & 红外相机 \\
\hline 金猫 & Pardofelis temmincktt & Carnivora & Felidae & II & 红外相机 \\
\hline 豹猫 & Prionailurus bengalensis & Carnivora & Felidae & & 红外相机 \\
\hline 金钱豹 & Panthera pardus & Carnivora & Felidae & I & 红外相机 \\
\hline 野猪 & Sus scrofa & Artiodactyla & Suidae & & 红外相机、样线 \\
\hline 林鹿 & Moschus berezovskii & Artiodactyla & Moschidae & I & 红外相机 \\
\hline 毛冠鹿 & Elaphodus cephalophus & Artiodactyla & Cervidae & & 红外相机 \\
\hline 小鹿 & Muntiacus reevesi & Artiodactyla & Cervidae & & 红外相机 \\
\hline 秦岭羚牛 & Budorcas bedfordi & Artiodactyla & Bovidae & I & 红外相机、样线 \\
\hline 中华斑羚 & Naemorhedus griseus & Artiodactyla & Bovidae & & 红外相机 \\
\hline
\end{tabular}


颜文博, 吉晟男, 帅凌鹰, 赵雷刚, 朱大鹏, 曾治高. 秦岭南坡陕西洋县辖区哺乳动物物种多样性的空间分 布格局. 生物多样性, 2019, 27 (2): 177-185.

http://www.biodiversity-science.net/CN/10.17520/biods.2018266

\begin{tabular}{|c|c|c|c|c|c|}
\hline 中文名 & 学名 & 目 & 科 & 保护等级 & 记录方式 \\
\hline Chinese name & Latin name & Order & Family & $\begin{array}{l}\text { Protection } \\
\text { level }\end{array}$ & Recording \\
\hline 中华瓺羚 & Capricornis milneedwardsii & Artiodactyla & Bovidae & II & 红外相机 \\
\hline 珀氏长吻松鼠 & Dremomys pernyi & Rodentia & Sciuridae & & 红外相机、样线 \\
\hline 岩松鼠 & Sciurotamias davidianus & Rodentia & Sciuridae & & 红外相机、样线 \\
\hline 隐纹花松鼠 & Tamias swinhoei & Rodentia & Sciuridae & & 红外相机、样线 \\
\hline 复齿鼠吾鼠 & Trogopterus xanthipes & Rodentia & Sciuridae & & 红外相机 \\
\hline 灰头小鼠鼠 & Petaurista caniceps & Rodentia & Sciuridae & & 红外相机 \\
\hline 大仓鼠 & Tscheskia triton & Rodentia & Cricetidae & & 夹捕 \\
\hline 甘肃仓鼠 & Cansumys canus & Rodentia & Cricetidae & & 夹捕 \\
\hline 秦岭鼠鼠 & Myospalax rufescens & Rodentia & Cricetidae & & 夹捕 \\
\hline 苛岗线鼠 & Caryomys inez & Rodentia & Cricetidae & & 夹捕 \\
\hline 滇攀鼠 & Vernaya fulva & Rodentia & Muridae & & 夹捕 \\
\hline 大林姬鼠 & Apodemus peninsulae & Rodentia & Muridae & & 夹捕 \\
\hline 黑线姬鼠 & A. agrarius & Rodentia & Muridae & & 夹捕 \\
\hline 齐氏姬鼠 & A. chevrieri & Rodentia & Muridae & & 夹捕 \\
\hline 黄胸鼠 & Rattus tanezumi & Rodentia & Muridae & & 夹捕 \\
\hline 褐家鼠 & R. norvegicus & Rodentia & Muridae & & 夹捕 \\
\hline 北社鼠 & Niviventer confucianus & Rodentia & Muridae & & 夹捕 \\
\hline 白腹巨鼠 & Leopoldamys edwardsi & Rodentia & Muridae & & 夹捕 \\
\hline 针毛鼠 & Niviventer fulvescens & Rodentia & Muridae & & 夹捕 \\
\hline 小家鼠 & Mus musculus & Rodentia & Muridae & & 夹捕 \\
\hline 中华竹鼠 & Rhizomys sinensis & Rodentia & Rhizomyidae & & 样线 \\
\hline 马来豪猪 & Hystrix brachyura & Rodentia & Hystricidae & & 红外相机、样线 \\
\hline 蒙古兔 & Lepus tolai & Lagomorpha & Leporidae & & 样线 \\
\hline
\end{tabular}

